\title{
Successful treatment of invasive pulmonary aspergillosis caused by Aspergillus felis, a cryptic species within the Aspergillus section Fumigati: A case report
}

Kazushi Yamairi, Kentaro Ido, Shigeki Nakamura, Makoto Niki, Waki Imoto, Wataru Shibata, Hiroki Namikawa, Hiroki Fujimoto, Koichi Yamada, Hirohisa Nakamae, Masayuki Hino, Yukihiro Kaneko, Yoshitsugu Miyazaki, Hiroshi Kakeya

\begin{tabular}{|c|l|}
\hline Citation & Journal of Infection and Chemotherapy, 25(4); 307-310 \\
\hline Issue Date & $2019-04$ \\
\hline Type & Journal Article \\
\hline Textversion & author \\
\hline Rights & $\begin{array}{l}\text { C2018 Japanese Society of Chemotherapy and The Japanese Association for Infectious } \\
\text { Diseases. This manuscript version is made available under the CC-BY-NC-ND 4.0 License. } \\
\text { https://creativecommons.org/licenses/by־nc-nd/4.0/ } \\
\text { This is the accepted manuscript version. Please cite only the published version. The article } \\
\text { has been published in final form at https://doi.org/10.1016/j.jiac.2018.10.016 }\end{array}$ \\
\hline DOI & \begin{tabular}{l}
$10.1016 / j . j i a c .2018 .10 .016$ \\
\hline
\end{tabular} \\
\hline
\end{tabular}

Self-Archiving by Author(s)

Placed on: Osaka City University

YAMAIRI, K., et al. (2019). Successful treatment of invasive pulmonary aspergillosis caused by Aspergillus felis, a cryptic species within the Aspergillus section Fumigati: A case report. Journal of Infection and Chemotherapy. 25, 307-310. doi: 10.1016/j.jiac.2018.10.016 
Successful treatment of invasive pulmonary aspergillosis caused by Aspergillus felis, a cryptic species within the Aspergillus section Fumigati: A case report

\author{
Kazushi Yamairi a,b, Kentaro Ido ${ }^{\text {c }}$, Shigeki Nakamura ${ }^{\text {d }}$, Makoto Niki e, Waki Imoto \\ a,b, Wataru Shibata ${ }^{\text {a,b }}$, Hiroki Namikawa ${ }^{\text {a,b }}$, Hiroki Fujimoto ${ }^{\text {a,b }}$, Koichi Yamada ${ }^{\text {a,b }}$, \\ Hirohisa Nakamae ${ }^{\text {c }}$, Masayuki Hino c , Yukihiro Kaneko ${ }^{\text {b,e }}$, Yoshitsugu Miyazaki d,
} Hiroshi Kakeya ${ }^{\text {a,b }}$

a Department of Infection Control Science, Osaka City University Graduate School of Medicine, 1-4-3, Asahi-machi, Abeno-ku, Osaka, 545-8585, Japan

b Research Center for Infectious Disease Sciences (RCIDS), Osaka City University Graduate School of Medicine, 1-4-3, Asahi-machi, Abeno-ku, Osaka, 545-8585, Japan

c Department of Hematology, Osaka City University Graduate School of Medicine, 1-43, Asahi-machi, Abeno-ku, Osaka, 545-8585, Japan

d Department of Chemotherapy and Mycoses, National Institute of Infectious

Diseases, 1-23-1, SHINJUKU-KU, Tokyo, 162-8640, Japan.

e Department of Bacteriology, Osaka City University, Graduate School of Medicine, 1-43, Asahi-machi, Abeno-ku, Osaka, 545-8585, Japan 
All authors meet the ICMJE authorship criteria.

Corresponding author: Hiroshi Kakeya, MD, PhD

Department of Infection Control Science, Osaka City University, Graduate School of Medicine

1-4-3, Asahi-machi, Abeno-ku, Osaka, 545-8585, Japan

Telephone: +81-06-6645-3784

Facsimile: $+81-06-6646-6056$

E mail: kakeya-ngs@umin.ac.jp 


\section{Abstract}

Aspergillus species are a major cause of life-threatening infections in immunocompromised hosts, and the most common pathogen of invasive aspergillosis is Aspergillus fumigatus. Recently, the development of molecular identification has revealed cryptic Aspergillus species, and A. felis is one such species within the Aspergillus section Fumigati reported in 2013.

We describe a case of invasive pulmonary aspergillosis caused by A. felis in a 41-yearold Japanese woman diagnosed with myelodysplastic syndrome. She presented with fever 19 days after undergoing autologous peripheral blood stem cell transplantation and was clinically diagnosed with invasive pulmonary aspergillosis. Bronchoscopy and bronchoalveolar lavage were performed for definitive diagnosis. The $\beta$-tubulin genes of the mold isolated from the bronchoalveolar lavage fluid, and sequenced directly from the PCR products using a primer pair were found to have $100 \%$ homology with A. felis. We successfully treated the patient with echinocandin following careful susceptibility testing.

To the best of our knowledge, this is the first published case reporting the clinical course for diagnosis and successful treatment of invasive aspergillosis by A. felis. 
Keywords: Aspergillus felis, Invasive aspergillosis, Cryptic species, myelodysplastic syndrome. 


\section{Introduction}

Invasive aspergillosis (IA) is a major problem in hematologic malignancies and hematopoietic cell transplantation [1]. The most common pathogen of IA is Aspergillus fumigatus, followed by $A$. niger, A. flavus, and A. terreus.

Recently, the development of molecular identification has revealed cryptic Aspergillus species that are very closely related and almost indistinguishable by morphologic methods [2, 3]. Some cryptic Aspergillus species are resistant and require higher minimum inhibitory concentrations (MIC) of azoles or amphotericin B deoxycholate, which are considered as first-line therapy for IA [4]. A. felis is one of the cryptic species within the Aspergillus section Fumigati described in 2013 [5]. It is known to cause IA in cats, dogs, and humans. There are few case reports of IA caused by cryptic Aspergillus species, especially A. felis.

Here, we report a clinical case of invasive pulmonary aspergillosis caused by $A$. felis that was successfully treated. 


\section{Case Report}

The patient was a 41-year-old Japanese woman diagnosed with myelodysplastic syndrome. She underwent autologous peripheral blood stem cell transplantation and had started taking fluconazole (FLCZ) to prevent fungal infection 4 months before as a part of transplantation conditioning. Neutrophil engraftment occurred on day 20 after transplantation. On day 19 after transplantation and 162 days of neutropenia (less than 500 neutrophils per microliter), she developed a fever. She tested positive for Aspergillus antigen, and thoracic computed tomography (CT) image showed an air crescent sign, suggesting invasive pulmonary aspergillosis in the lung on day 27 after transplantation (Figure 1). We clinically diagnosed her with invasive pulmonary aspergillosis, and initially started intravenous treatment with voriconazole (VRCZ) $(6 \mathrm{mg} / \mathrm{kg}$ twice daily for 2 days, followed by $4 \mathrm{mg} / \mathrm{kg}$ every $12 \mathrm{~h}$ ). Bronchoscopy and bronchoalveolar lavage (BAL) was performed for definitive diagnosis on day 37 after transplantation.

There was no change in the lung lesion after one month, and the fever persisted (Figure 1). Considering the low voriconazole trough blood level, we switched to treatment with liposomal amphotericin-B (L-AMB) $(2.5 \mathrm{mg} / \mathrm{kg}$ per day). However, she developed nausea as a side effect, and therefore, L-AMB therapy was stopped after one week. Subsequently, we reintroduced combination therapy with VRCZ (4 mg/kg every $12 \mathrm{~h})$ and 
caspofungin (CPFG) (70 mg loading dose, followed by $50 \mathrm{mg}$ daily). The lesion gradually decreased in size.

Filamentous fungi were isolated from the bronchoalveolar lavage fluid (BAL) on day 10 of the culture. Compared to A. fumigatus, white colonies of the isolates grew slowly, and there was no morphologically special point (Figure 2). The $\beta$-tubulin genes of the mold isolated from BAL were sequenced directly from the PCR products using a primer pair published previously for species identification [6]. The sequence showed $100 \%$ similarity with A. felis genes, using the BLAST database (www.ncbi.nlm.nih.gov/BLAST).

Susceptibility testing was performed according to the recommendations of the Clinical and Laboratory Standard Institute (CLSI) document M38-A2 [7]. The MICs for amphotericin B, VRCZ, itraconazole, and FLCZ were found to be 2.0, > 8.0, > 8.0, and $64 \mu \mathrm{g} / \mathrm{ml}$, respectively (Table 1). The minimal effective concentration (MEC) for micafungin (MCFG) was $<0.015 \mu \mathrm{g} / \mathrm{ml}$ (Table 1). CPFG was not tested.

We stopped treatment with VRCZ and CPFG and switched to a single dose of MCFG (150 mg per day) based on its low MEC value. We continued to treat her as an outpatient successfully for a total of 5 months.

\section{Discussion}


Aspergillus species are a major cause of life-threatening infections in

immunocompromised hosts, including patients with hematologic malignancies and those undergoing hematopoietic cell transplantation [1]. Studies have revealed the presence of cryptic Aspergillus species in clinical samples using advanced genetic identification technology. In a recent study, over $10 \%$ of all aspergilli clinically isolated were cryptic species $[8,9]$.

A. felis, an emerging causative agent of invasive aspergillosis in humans, is one of the cryptic Aspergillus species in the section Fumigati and was genotypically identified as a new species in 2013 [5]. Only two human cases of IA caused by $A$. felis have been published so far. These cases, which were initially reported to be caused by A. viridinutans were subsequently attributed to A. felis following a reinvestigation [10-12]. However, both patients died during the course of the treatment. To the best of our best knowledge, this is the first reported case of invasive pulmonary aspergillosis caused by A. felis which has been treated successfully.

Like some other drug-resistant cryptic Aspergillus species, A. felis also requires higher MICs of azoles including VRCZ, which are considered the first-line therapy for IA [13]. Additionally, multi-azole resistant cases of $A$. felis such as this one have been reported [5]. Previous studies have reported amphotericin B to have MICs of $\leqq 1 \mu \mathrm{g} / \mathrm{ml}$ 
in A. felis $[5,10,11]$. However, we found that the MIC of amphotericin B for A. felis, in this case, was $2 \mu \mathrm{g} / \mathrm{ml}$, which is relatively high. Additionally, we could not determine the clinical efficacy of liposomal amphotericin B (L-AMB) since we used it only for a short time due to side effects. High MICs of amphotericin B are required for some resistant cryptic Aspergillus species, which have been associated with poor clinical outcomes [2, 5, 14-16]. Previous studies have reported relatively low MECs of echinocandin for $A$. felis. Especially, the MEC of MCFG was lower than that of the CPFG [5]. The regression of the lung lesion in response to echinocandin, in this case, suggests that echinocandin is effective against $A$. felis.

However, clinical data on the treatment of cryptic Aspergillus species including A. felis is limited. Hence, an effective treatment for invasive aspergillosis caused by cryptic Aspergillus species is not well established [17]. In this case, the combination of antifungal treatments using VRCZ plus CPFG was effective. Though combination therapies seem attractive, there is no data available to confirm their effectiveness [17]. Furthermore, in the two cases of IA reported previously, A. felis was refractory to combination antifungal treatments using VRCZ plus CPFG and L-AMB plus CPFG respectively, and the outcomes were fatal in both cases $[9,10]$. Therefore, for a treatment to be successful, it is important to determine the exact nature of the underlying disease in 
order to administer the right dose of appropriate antifungal agents [17].

Since susceptibility to antifungal agents varies largely among different fungal isolates and species $[18,19]$, it is important to consider the choice of these agents based on the correct identification of the fungal species and their susceptibilities.

In Japan, there are only a few institutions that have the capability to identify fungal species and antifungal susceptibility accurately. It is, therefore, necessary to maintain core facilities that can help with this identification process. 


\section{Acknowledgements}

This research was supported by the Research Program on Emerging and

Re-emerging Infectious Diseases from the Japan Agency Development,

AMED [Grant number JP17fk0108208 and 18fk0108052h0002] and JSPS

KAKENHI [Grant number 16K09939].

Conflict of interest

Hiroshi Kakeya has received grant support from Pfizer Japan Inc., MSD KK,

Astellas Pharma Inc., Dainippon Sumitomo Pharma Co., Ltd. 


\section{References}

[1] Kontoyiannis DP, Marr KA, Park BJ, Alexander BD, Anaissie EJ, Walsh TJ, et al. Prospective surveillance for invasive fungal infections in hematopoietic stem cell transplant recipients, 2001-2006: overview of the Transplant-Associated Infection Surveillance Network (TRANSNET) database. Clin Infect Dis 2010; 50:1091-100.

[2] Balajee SA, Nickle D, Varga J, Marr KA. Molecular studies reveal frequent misidentification of Aspergillus fumigatus by morphotyping. Eukaryot Cell 2006; 5: $1705-12$.

[3] Montenegro G, Puch SS, Jewtuchowicz VM, Pinoni MV, Relloso S, Temporitti E, et al. Phenotypic and genotypic characterization of Aspergillus lentulus and Aspergillus fumigatus isolates in a patient with probable invasive aspergillosis. J Med Microbiol 2009; 58: 391-5.

[4] Howard SJ. Multi-resistant aspergillosis due to cryptic species. Mycopathologia $2014 ; 178: 435-9$.

[5] Barrs VR, van Doorn TM, Houbraken J, Kidd SE, Martin P, Pinheiro MD, et al. Aspergillus felis sp. nov.an emerging agent of invasive aspergillosis in humans, cats, and dogs. PLoS One 2013; 14; 8:e64871. 
[6] Balajee SA, Gribskov JL, Hanley E, Nickle D, Marr KA. Aspergillus lentulus sp. nov., a new sibling species of A. fumigatus. Eukaryot Cell 2005; 4:625-32.

[7] Clinical and Laboratory Standards Institute (CLSI). Reference method for broth dilution antifungal susceptibility testing of filamentous fungi: approved standard M38A2. 2nd ed. Wayne, PA: Clinical and Laboratory Standards Institute; 2008.

[8] Alastruey-Izquierdo A, Mellado E, Peláez T, Pemán J, Zapico S, Alvarez M, et al. Population-based survey of filamentous fungi and antifungal resistance in Spain (FILPOP Study). Antimicrob Agents Chemother 2013; 57:3380-7.

[9] Balajee SA, Kano R, Baddley JW, Moser SA, Marr KA, Alexander BD, et al. Molecular identification of Aspergillus species collected for the Transplant-Associated Infection Surveillance Network. J Clin Microbiol 2009; 47:3138-41.

[10] Coelho D, Silva S, Vale-Silva L, Gomes H, Pinto E, Sarmento A, et al. Aspergillus viridinutans: an agent of adult chronic invasive aspergillosis. Med Mycol 2011; 49:7559.

[11] Pelaez T, Alvarez-Perez S, Mellado E, Serrano D, Valerio M, Blanco JL. Invasive aspergillosis caused by cryptic Aspergillus species: a report of two consecutive episodes in a patient with leukaemia. J Med Microbiol 2013; 62:474-8. 
[12] Alvarez-Pérez S, Mellado E, Serrano D, Blanco JL, Garcia ME, Kwon M, et al.

Polyphasic characterization of fungal isolates from a published case of invasive aspergillosis reveals misidentification of Aspergillus felis as Aspergillus viridinutans. $\mathrm{J}$ Med Microbiol 2014; 63:617-9.

[13] Van Der Linden JWM, Warris A, Verweij PE. Aspergillus species intrinsically resistant to antifungal agents. Med Mycol 2011; 49: S82-9.

[14] Alcazar-Fuoli L, Mellado E, Aslastruey-Izquierdo A, Cuenca-Estrella M, Rodriguez-Tudela JL. Aspergillus section Fumigati: antifungal susceptibility patterns and sequence-based identification. Antimicrob Agents Chemother 2008; 52:1244-51. [15] Vinh DC, Shea YR, Sugui JA, Parrilla-Castellar ER, Freeman AF, Campbell JW, et al. Invasive aspergillosis due to Neosartorya udagawae. Clin Infect Dis 2009; 49:10211.

[16] Lass-Florl C, Griff K, Mayr A, Petzer A, Gastl G, Bonatti H, et al. Epidemiology and outcome of infections due to Aspergillus terreus: 10-year single centre experience. Br J Haematol 2005; 131:201-7.

[17] Nedel WL, Pasqualotto AC. Treatment of infections by cryptic Aspergillus species. Mycopathologia 2014; 178:441-5.

[18] Alastruey-Izquierdo A, Alcazar-Fuoli L, Cuenca-Estrella M. Antifungal 
susceptibility profile of cryptic species of Aspergillus. Mycopathologia 2014; 178:42733.

[19] Howard SJ. Multi-resistant aspergillosis due to cryptic species. Mycopathologia $2014 ; 178: 435-9$. 
Table 1. Susceptibility testing of a clinical isolate of $A$. felis based on the recommendations of the Clinical and Laboratory Standard Institute (CLSI) document M38-A2

\begin{tabular}{ccccccc}
\hline & MCFG $^{* *}$ & AMPH $^{*}$ & $5-\mathrm{FC}^{*}$ & $\mathrm{FLCZ}^{*}$ & $\mathrm{ITCZ}^{*}$ & $\mathrm{VRCZ}^{*}$ \\
\hline A. felis & $0.015>$ & 2 & $>64$ & $>64$ & $>8$ & $>8$ \\
\hline
\end{tabular}

*MIC, minimum inhibitory concentration * "MEC, minimum effective concentration MCFG, micafungin; AMPH, Amphotericin B; 5-FC,5-fluorocytosine; FLCZ, fluconazole; ITCZ, itraconazole; VRCZ, voriconazole. 


\section{Figure legends}

Figure 1 . The clinical course used to treat invasive pulmonary aspergillosis caused by Aspergillus felis. VRCZ: voriconazole, L-AMB: liposomal amphotericin B, CPFG:

caspofungin, MCFG: micafungin

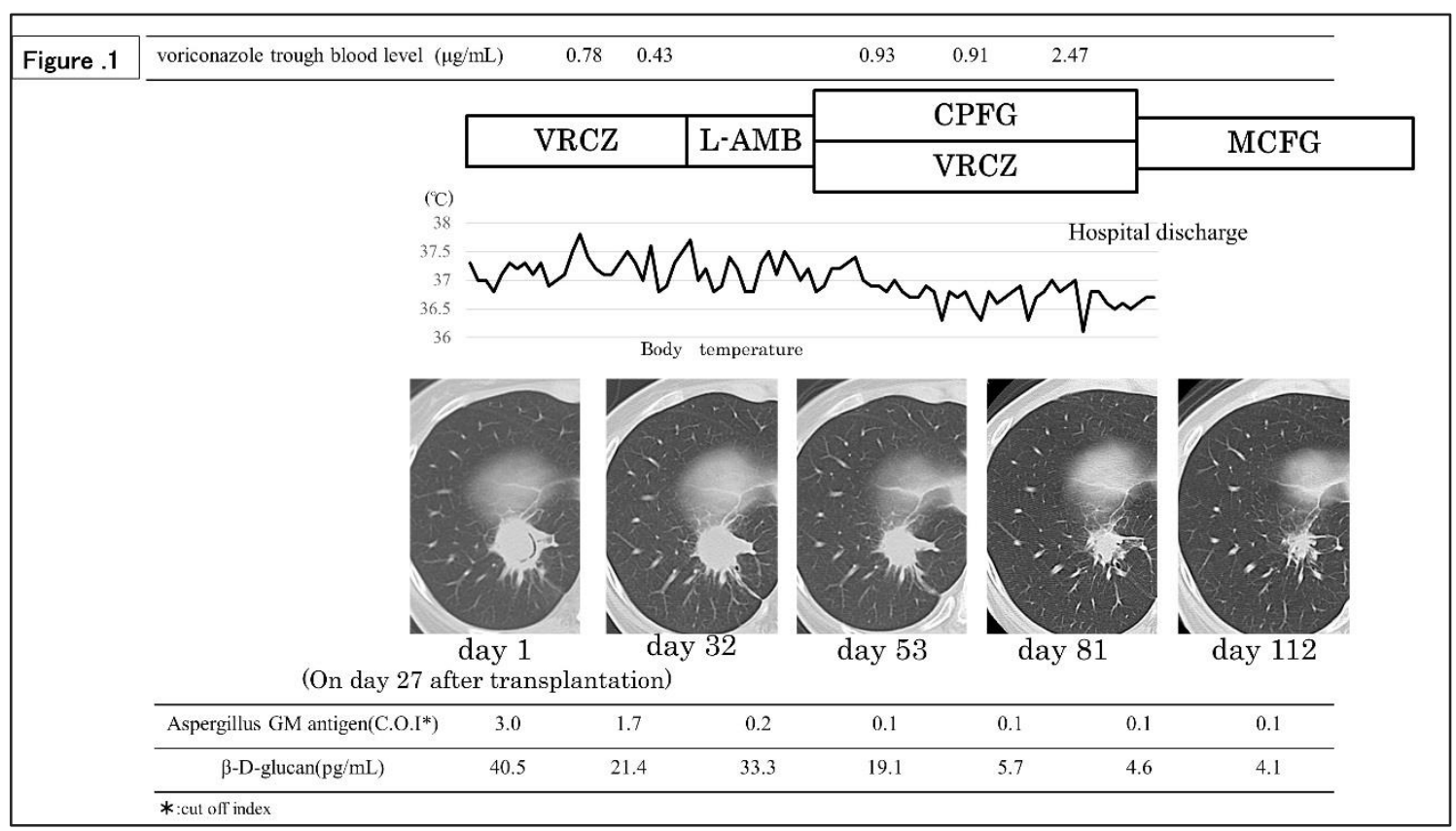


Figure 2. Aspergillus culture. (A) Image of Aspergillus felis culture in Sabouraud

Dextrose agar at $30^{\circ} \mathrm{C}$ on day 9. (B) Microscopical examination of lactophenol cotton

blue stain of Aspergillus felis.

\section{Figure.2}
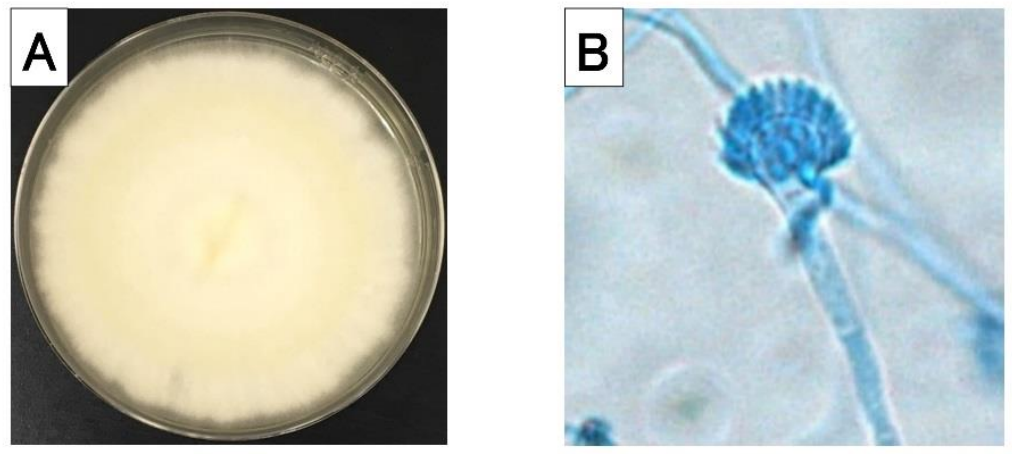